\title{
Application of Hybrid Real-Time Simulation Concept for Modeling of HVDC Back-to-Back Link
}

\author{
Vladimir Rudnik ${ }^{1, a)}$, Ruslan Ufa ${ }^{1, \text { b) }}$ and Alexey Maltsev ${ }^{1, c)}$ \\ ${ }^{1}$ National Research Tomsk Polytechnic University, 634050 Tomsk, Russia. \\ ${ }^{a)}$ Corresponding author: fordlp006@mail.ru \\ b) hecn@tpu.ru \\ c)maltsevaleksey@mail.ru
}

\begin{abstract}
The application of new technologies considerably complicates the structure and the configuration of the electric grids, changes its dynamic proper-ties and creates new challenges to power system planning and operation for providing reliable and efficient use of intelligent electrical power systems. Considering of well-known specificity of electrical power systems operation, the modelling is the main way of effective solution of the problems. Hybrid simulation method is chosen as the one of the most appropriate approach. Adequate modeling of HVDC devices defines the inadmissible of separate simulation of different modes in equipment and electrical grids as a whole. It is proves by carried out research of hybrid simulation technique witch combine the different simulation approaches.
\end{abstract}

\section{INTRODUCTION}

Nowadays there are the trends of increasing in the number of pro-jects connected with the intelligent electrical power systems (EPS) based on the power semiconductor devices for the High voltage direct current transmission system (HVDC).

The application of new technologies considerably complicates the structure and the configuration of the EPS, changes its dynamic proper-ties and creates new challenges to power system planning and operation for providing reliable and efficient use of intelligent EPS.

The most complex challenges are among [1, 2, 3]:

- Analysis of the influence of FACTS and HVDC devices, their control and regulation systems on the power equipment and alternating current $(\mathrm{AC})$ power system in the whole;

- Debugging of control systems for such devices.

Mentioned tasks become more urgent from the point of view of planned for implementation the HVDC projects in Russia, particularly the application of HVDC back-to-back link (B2B) based on voltage source convector (VSC).

Considering of well-known specificity of EPS operation, the simulation remains the main tool for effective problems solution. Hybrid simulation method is chosen as the one of the most appropriate approach.

\section{THE OPERATION SPECIFICITY OF THE HVDC DEVICES BASED ON VSC}

The operation specificity of the HVDC devices based on VSC should be taken into account during development of its models:

- $\quad$ Phase-to-phase operation;

- Application of high-speed fully-controlled power semiconductor switches;

- Continuous operation in quasi-steady state and transients processes.

Proceeding from the aforesaid, comprehensive simulation of mentioned HVDC devices defines the inadmissible of separate simulation of electromagnetic and electromechanical transient processes in electric grids. This statement 
is confirmed by observed trends in research and development of hybrid simulation tools, based on application of different simulation approaches.

The main idea of the hybrid approach are [4, 5]:

- to describe all power equipment in EPS the corresponding mathematical models, which adequately represented the main spectrum of normal and abnormal modes in power equipment and EPS as a whole, are used;

- $\quad$ to describe all power equipment in EPS, which include the switching elements, the corresponding model physical models are used;

- the connection between the math and physic models is provided by using of appropriate voltage-current converters;

- $\quad$ to provide all the functions of information and control, the digital level are used to realized it, in particular modeling of the systems of control and protection is realized via application a digital-to-analog, analog-to-digital transformation and a specialized client and server software

The basis of Hybrid real-time simulator (HRTSim) of EPS is formed by specialized hybrid processors (SHP), in which each equipment of a simulated EPS is reproduced: an electric machine, a transformer, a load, etc. In the joint venture, all information and control functions are per-formed by a microprocessor unit (MPU), a hybrid coprocessor (HCP) performs the solution of EPS equipment mathematical models, and voltage / current converters (VCC) match physical currents to mathematical variables, allowing the physic level to combine joint ventures in accordance with the scheme of the modeled EPS when using switch of three-phase nodes (STPN).

According to the mentioned points of conception and analysis of HVDC B2B link operation principle the structural schemes the HVDC B2B model was developed and presented [4, 5, 6].

In Fig. 1 the dynamic monitoring and control panel is presented.

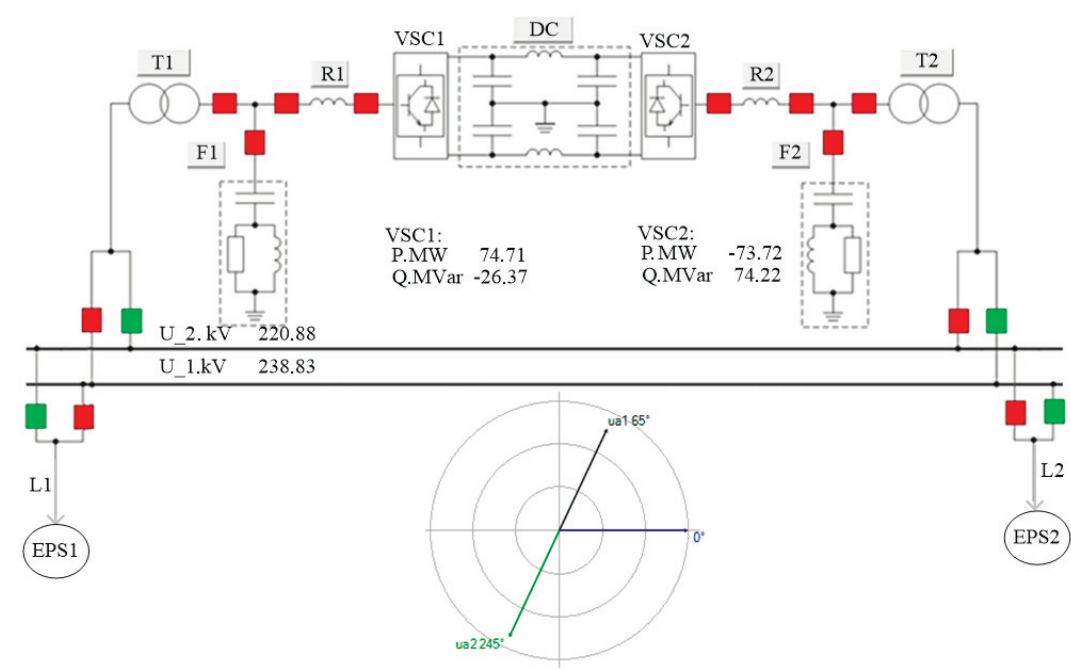

FIGURE 1. Dynamic monitoring and control panel of HVDC B2B link model

Figure 2 shows the oscillograms of power flow control through the HVDC B2B model. The results of the above modeling presents the processes of regulation and reverse power flow: changes in power flow, accompanied by changes in the voltage level on a capacitor bank of a DC circuit and reactive power. Figure 3 shows the oscillograms of asynchronous interconnection via HVDC B2B model under the theoretically the worst mode, than angel between voltages is $\delta=180^{\circ}$. The process is not accompanied by deep synchronized swings and is not resynchronized the mode of EPS. Therefore, the use of HVDC B2B link is the most preferable for a guaranteed reliable solution of asynchronous interconnection. 


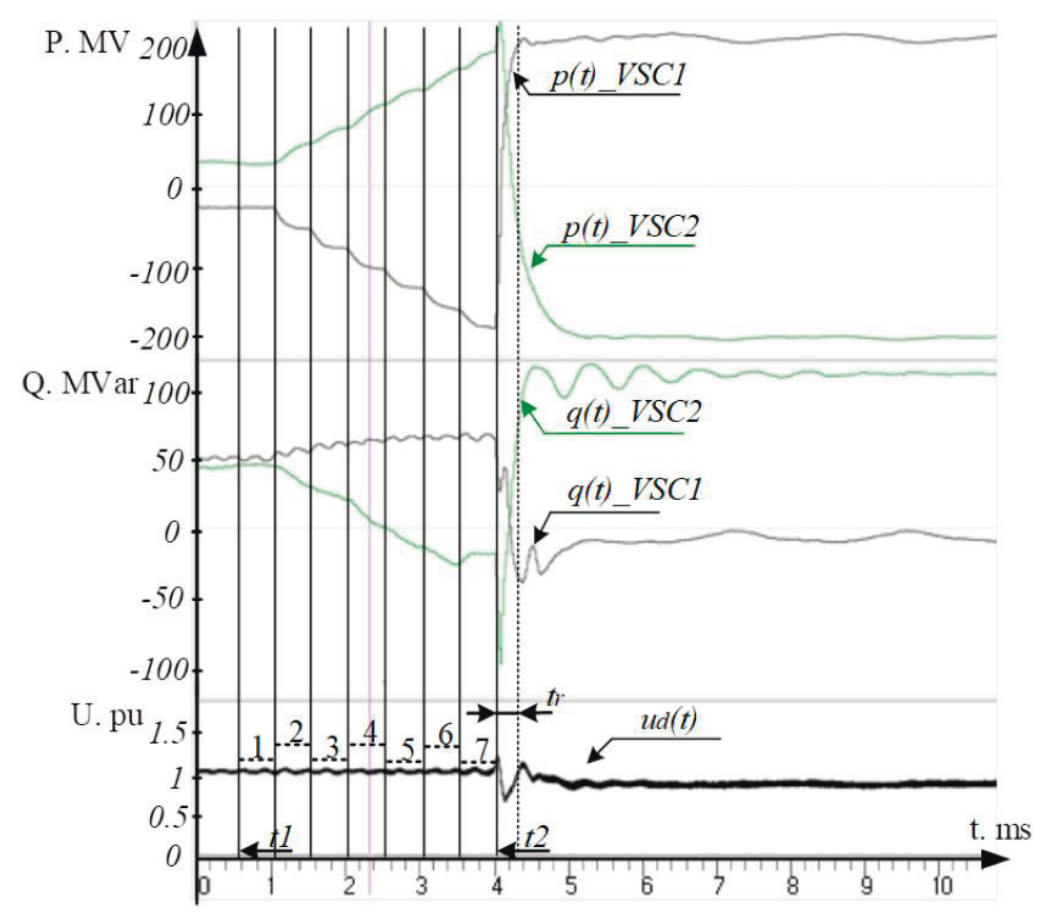

FIGURE 2. The processes of regulating the flow of power through the B2B

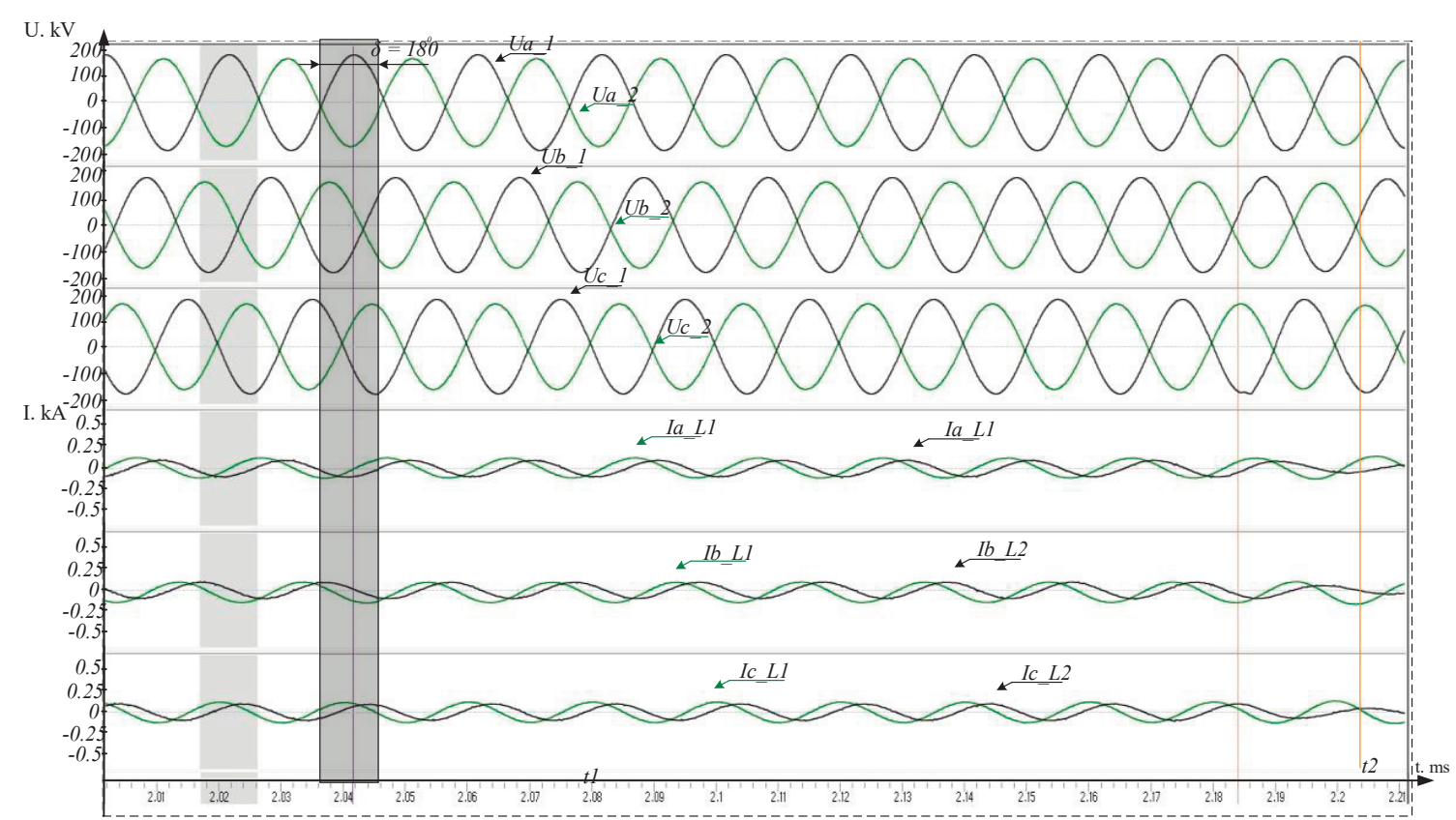

FIGURE 3. The processes of asynchronous interconnection via HVDC B2B model under the theoretically the worst mode, than angel between voltages is $\delta=180^{\circ}$

\section{CONCLUSION}

The application of the proposed concept allows obtaining the following key benefits:

- $\quad$ Real time simulations in unlimited range; 
- Interconnection with any special devices for closed-loop testing and adjustment of control and protection system;

- Simulation of full spectrum of electromagnetic and electromechanical transient processes in electric grids for analysis tasks of mutual influence of HVDC devices and AC.

The presented results of modeling of process of regulation and reverse power flow, and asynchronous interconnection via HVDC B2B model are shown the adequacy of the model and confirms the possibility of using the HVDC B2B link to effectively solve the above task.

\section{ACKNOWLEDGMENTS}

The reported study was funded by RFBR according to the research project No 18-38-00862.

\section{REFERENCES}

1. D. Qi, Electra, 2574-16, 12 (2011).

2. Z. Jiebei and C. Booth, International Universities Power Engineering Conference (UPEC 2010, Cardiff, 2010), pp.1-6.

3. M. V. Andreev, A. S. Gusev, N. Yu. Ruban, A. A. Suvorov, R. A. Ufa, A. B. Askarov, J. Bemš and T. Králík, IEEE Transactions on Power Syst, 34(2), 1404-1415, (2019).

4. A. A. Suvorov, Gusev A.S., A.O. Sulaymanov and O.V. Suslova, MATEC Web Conf. 91, 1-4 ( 2017).

5. A. Suvorov, M. Andreev, N. Ruban and R. Ufa, IEEE PES Innovative Smart Grid Technologies Conference Europe (ISGT Europe, Torino, 2017), pp. 1-6.

6. M. Andreev, Y. Borovikov, A. Gusev, A. Sulaymanov, N. Ruban, A. Suvorov, R. Ufa, J. Bemš and T. Králík, IET Gener, Transmission and Distrib. 12, 133-141 (2018). 